\title{
Activation Stress for Slip Systems of Pure Magnesium Single Crystals in Pure Shear Test ${ }^{* 1}$
}

\author{
Kazutaka Fukuda $^{1,{ }^{* 2}}$, Yuta Koyanagi ${ }^{1,{ }^{* 2}}$, Masayuki Tsushida ${ }^{2}$, Hiromoto Kitahara ${ }^{3}$, Tsuyoshi Mayama ${ }^{4}$ \\ and Shinji Ando ${ }^{5}$
}

${ }^{1}$ Graduate School of Science and Technology, Kumamoto University, Kumamoto 860-8555, Japan

${ }^{2}$ Faculty of Engineering, Kumamoto University, Kumamoto 860-8555, Japan

${ }^{3}$ Institute of Pulsed Power Science, Kumamoto University, Kumamoto 860-8555, Japan

${ }^{4}$ Priority Organization for Innovation and Excellence, Kumamoto University, Kumamoto 860-8555, Japan

${ }^{5}$ Magnesium Research Center, Kumamoto University, Kumamoto 860-8555, Japan

With a hexagonal close packed structure, magnesium has many slip systems. Pure shear tests were carried out to evaluate their critical resolved shear stresses (CRSS). As a result, the CRSS for the basal slip was 0.7 MPa, and it was close to conventional values. When the shear stress of $40 \mathrm{MPa}$ was applied in parallel to the prismatic plane, the $\{10 \overline{1} 2\}$ twin deformation occurred only, and the prismatic slip deformation did not occur. [doi:10.2320/matertrans.M2016402]

(Received November 11, 2016; Accepted January 11, 2017; Published February 10, 2017)

Keywords: magnesium single crystal, basal slip, prismatic slip, twin, CRSS (critical resolved shear stresses)

\section{Introduction}

Magnesium (Mg) and its alloys are attractive candidate materials for use in lightweight technology due to their light weight and high specific strength. However, Mg shows low ductility at room temperature. Investigation is required to determine deformation mechanisms in order to improve such mechanical properties. Mg with a hexagonal close-packed (hcp) structure has various deformation mechanisms. There are four slip systems in hcp metals: $(0001)\langle 11 \overline{2} 0\rangle$ basal slip ${ }^{1)}$, $\{10 \overline{1} 0\}\langle 11 \overline{2} 0\rangle$ prismatic slip ${ }^{2)},\{10 \overline{1} 1\}\langle 11 \overline{2} 3\rangle$ first order pyramidal slip ${ }^{2)}$, and $\{11 \overline{2} 2\}\langle\overline{1} \overline{1} 23\rangle$ second order pyramidal slip ${ }^{3)}$. It is known that the basal slip is the main slip system in $\mathrm{Mg}$, and the critical resolved shear stress (CRSS) has been reported to be approximately $0.5 \mathrm{MPa}^{4)}$. Here, in von-Mises' criterion $^{5)}$, five independent slip systems are required to deform a crystal to any arbitrary shape. Since the number of basal slips is insufficient for this criterion, it is clear that non-basal slips are also activated.

While Asada et ll $^{2)}$ have reported that non-basal slips were activated at temperatures above $393 \mathrm{~K}$ but not at room temperature in tensile tests of pure $\mathrm{Mg}$ single crystals, Ando et $a l .{ }^{3)}$ have also reported that pure magnesium single crystals yielded due to $\{11 \overline{2} 2\}$ pyramidal slips at temperatures between $77 \mathrm{~K}$ and $293 \mathrm{~K}$. It is thus clear that deformation mechanism in tensile tests of $\mathrm{Mg}$ single crystals remains unclarified. One reason for this is that, when a basal slip system with a low CRSS is activated in tensile or compression tests of $\mathrm{Mg}$ single crystals, investigation of other CRSSs for non-basal slip systems with different CRSSs is difficult. Shear test would be suitable to evaluate CRSSs for individual slip systems since shear force can be directly loaded to a slip plane. Miyauchi et $a l .{ }^{6)}$ have suggested a pure shear test; as described in their reference, four notches were symmetrically induced into steel

\footnotetext{
${ }^{* 1}$ This Paper was Originally Published in Japanese in J. Japan Inst. Met. Mater. 80 (2016) 334-339.

${ }^{* 2}$ Graduate Student, Kumamoto University
}

sheets, and the central part of the sheet was vertically pulled out. To expand on this, our study subjects single Mg crystals to the pure shear test and evaluates CRSSs for basal and non-basal slips.

\section{Experimental Procedures}

A $99.99 \%$ pure-magnesium ingot and seed crystal were installed in a graphite mold. Mg single crystals were grown by the modified Bridgeman method using a graphite mold in a high-purity argon gas atmosphere. The growth rate was $8.3 \times$ $10^{-3} \mathrm{~mm} / \mathrm{s}$. Mg single crystals were crystallographically analyzed by the X-ray back reflection Laue method and were then cut into sheet specimens by a non-distortion cutting machine using a stainless wire with a diameter of $0.5 \mathrm{~mm}$ and nitric acid. Surfaces of the specimens were chemically pol-

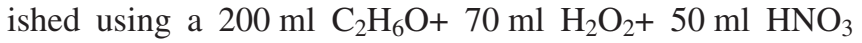
solution. Figure 1(a) shows schematic illustrations of (a) pure shear test specimen and (b) overview of the pure shear test method. The size of the specimen with four notches in symmetry was approximately $20 \times 20 \times 2 \mathrm{~mm}^{3}$. The specimens (a)

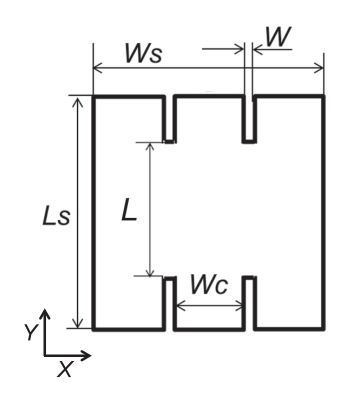

(b)

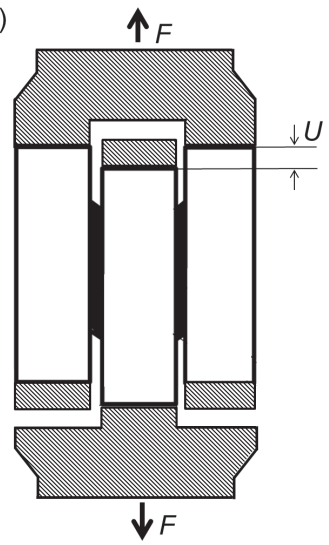

Fig. 1 Schematic illustrations of (a) pure shear test specimen and (b) pure shear test method. 
were subjected to thermal cyclic annealing both to decrease dislocation density and to remove any strains induced by cutting and polishing. For each cycle, the polished specimens were annealed for $21.6 \mathrm{Ks}$ between $673 \mathrm{~K}$ and $723 \mathrm{~K}$. A total of 8 annealing cycles were applied. Two kinds of $\mathrm{Mg}$ single crystals for the pure shear test were prepared to evaluate CRSSs for $\{0001\}\langle 11 \overline{2} 0\rangle$ basal and $\{10 \overline{1} 0\}\langle 11 \overline{2} 0\rangle$ prismatic slips. Figure 2 shows schematic illustrations of $\alpha$ and $\beta$ specimens used in this study. Shear directions in $\alpha$ and $\beta$ specimens were respectively parallel to $\{0001\}$ and $\{10 \overline{1} 0\}$; actual specimen sizes are summarized in Table 1.

A jig for the pure shear test was prepared and set to an Instron type universal testing machine. Pure shear tests were carried out at $298 \mathrm{~K}$ with a cross head speed of $1.67 \times$ $10^{-3} \mathrm{~mm} / \mathrm{s}$, as shown in Fig. 1(b). Shear stress, $\tau$ and shear strain, $\gamma$ were calculated from eqs. (1) and (2),

$$
\begin{gathered}
\tau=F /(2 * L * T) \\
\gamma=U / W
\end{gathered}
$$

where $F$ is the drawing force, $L$ is shear area length, $T$ is specimen thickness, $U$ is the shear displacement obtained from the testing machine, and $W$ is shear area width, as shown in Fig. 1. $U$ is the displacement obtained by subtracting the elastic displacement from the displacement of load-displacement curves. Slip line observations and displacement measurements in shear areas were carried out with an optical microscope by Nomarski differential interference contrast microscopy after the pure shear test. Stress distribution in pure shear test specimens was evaluated by the finite element method (FEM) with ANSYS software.
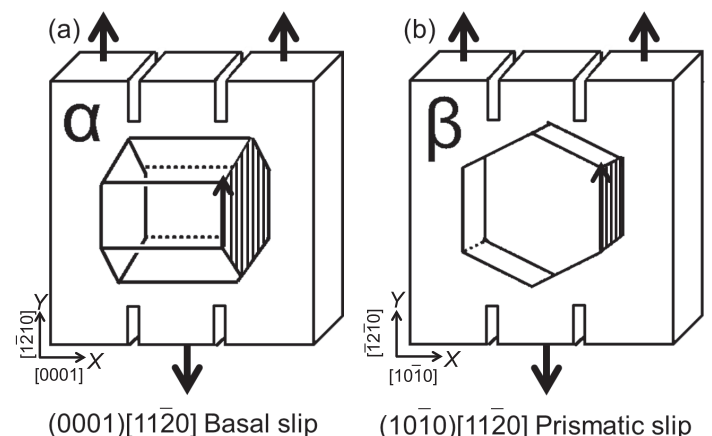

Fig. 2 Schematic illustrations of (a) $\alpha$ specimen and (b) $\beta$ specimen used in this study.

Table 1 Specimen sizes of pure shear test specimens used in this study.

\begin{tabular}{ccccccc}
\hline Specimen & $H / \mathrm{mm}$ & $L s / \mathrm{mm}$ & $T / \mathrm{mm}$ & $W / \mathrm{mm}$ & $L / \mathrm{mm}$ & $W c / \mathrm{mm}$ \\
\hline$\alpha 1$ & 22.03 & 18.75 & 1.41 & 1.14 & 7.58 & 6.73 \\
$\alpha 2$ & 22.02 & 19.35 & 1.86 & 1.10 & 11.58 & 6.74 \\
$\alpha 3$ & 26.14 & 19.35 & 1.94 & 0.94 & 11.33 & 6.73 \\
$\alpha 4$ & 24.76 & 19.45 & 2.03 & 0.92 & 10.88 & 6.46 \\
$\beta 1$ & 23.70 & 19.50 & 1.59 & 0.92 & 2.28 & 6.38 \\
$\beta 3$ & 23.70 & 19.60 & 1.70 & 1.03 & 1.53 & 6.45 \\
$\beta 4$ & 25.00 & 19.55 & 2.15 & 0.91 & 2.33 & 6.32 \\
\hline
\end{tabular}

\section{Results and Discussion}

\section{1 $\alpha$ specimen}

Figure 3 shows typical shear stress - displacement curve of $\alpha$ specimen. Shear yield stresses of $\alpha$ and $\beta$ specimens are summarized in Table 2. The shear yield stress of $\alpha$ specimen was approximately $0.7 \mathrm{MPa}$. Figure 4 shows optical micrographs of the shear area at (a) $U=0.01 \mathrm{~mm}$ and (b) $U=$ $0.41 \mathrm{~mm}$ in $\alpha$ specimen. $\alpha$ specimen yielded due to the basal slip operation; this is known as the slip bands parallel to the shear direction were clearly observed in the shear area at $U=$ $0.01 \mathrm{~mm}$. Also, the width of slip bands did not change at $U=$ $0.41 \mathrm{~mm}$, as shown in Fig. 4(b). Here, the shear displacement, $d$ was obtained by measuring movement of etch pits along the line $\mathrm{X}$ in Fig. 4(b). Etch pit formed during sample preparation before mechanical testing. Figure 5 shows the relationship between the position on line $\mathrm{X}$ through the shear area and shear displacement, $d$. As a result, shear deformation was found to have occurred uniformly within the slip band. Additionally, there was no displacement in the direction perpendicular to the shear direction in the shear area. These results indicate that only the basal slip was activated. Figure 6 shows relationships between the $U$ and $d$ of each $\alpha$ specimen, which are mostly identical, indicating that only the basal slip activated in the shear area of $\alpha$ specimen in the pure shear test.

CRSS for the basal slip was calculated using shear yield

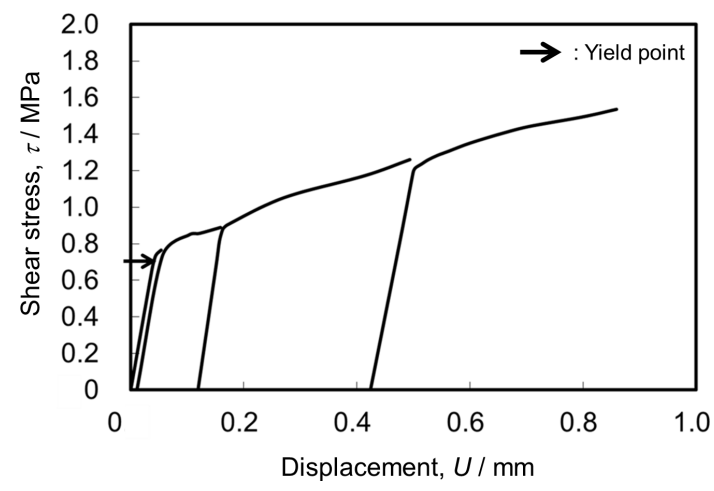

Fig. 3 Typical shear stress - displacement curve of $\alpha 1$ specimen.

Table 2 Shear yield stress of $\alpha$ and $\beta$ specimens.

\begin{tabular}{cccccccc}
\hline Specimen & $\alpha 1$ & $\alpha 2$ & $\alpha 3$ & $\alpha 4$ & $\beta 1$ & $\beta 3$ & $\beta 4$ \\
\hline Yield stress, $\sigma_{y} / \mathrm{MPa}$ & 0.7 & 0.6 & 0.7 & 0.7 & 7.3 & 10 & 5.5 \\
\hline
\end{tabular}

(a)

(b)

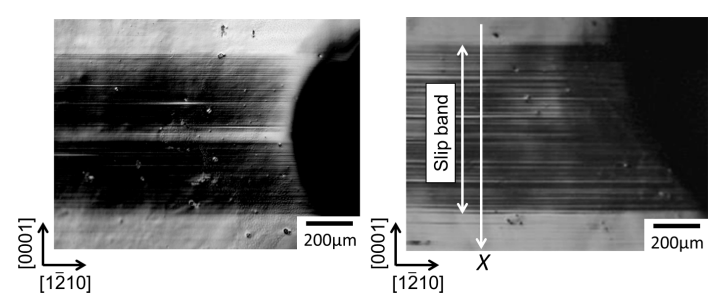

Fig. 4 Optical photographs of shear area in $\alpha$ specimen at (a) $U=0.01 \mathrm{~mm}$ and (b) $U=0.41 \mathrm{~mm}$. Slip bands of basal slip are clearly observed. 


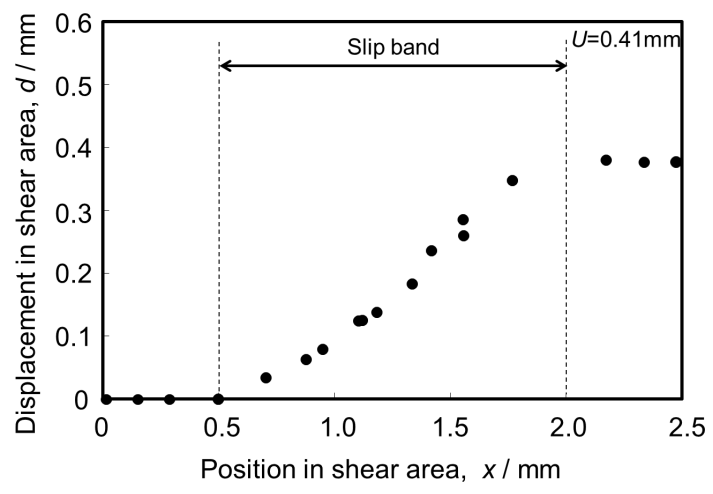

Fig. 5 Relationship between position on line $X$ shown in Fig. 4 (b) and shear displacement in shear area of $\alpha$ specimen at $U=0.41 \mathrm{~mm}$.

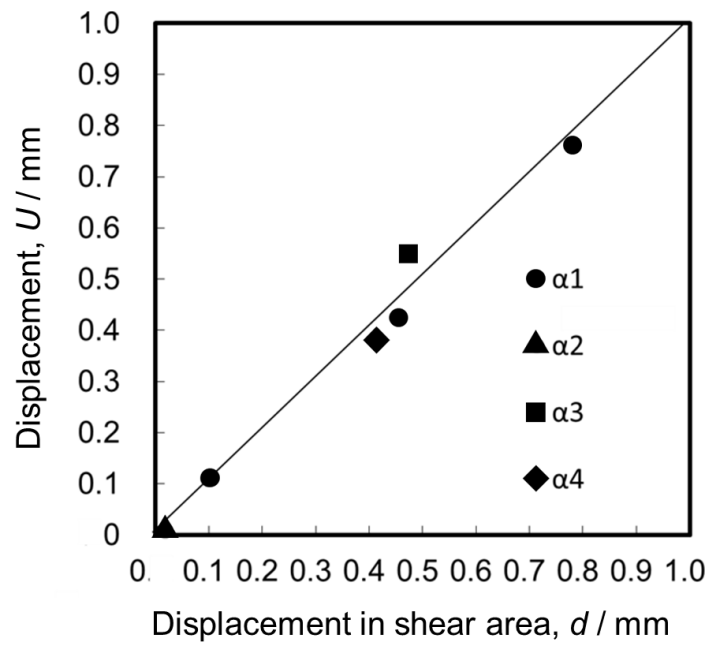

Fig. 6 Relationship between shear displacement $d$ obtained by measuring movement of etch pits along line X shown in Fig. 4 (b) and shear displacement $U$ obtained from testing machine.

stresses shown in Table 2. There are three kinds of basal slips, with the different slip directions in $\alpha$ specimen shown in Fig. 7: b1, b2 and b3. The basal slip, b1, would be the first activated in the pure shear test since its slip direction is parallel to the shear direction. Therefore, CRSS for the b1 basal slip corresponds to shear stress and is estimated to be $0.7 \mathrm{MPa}$. This value is close to the reported CRSS $(0.5 \mathrm{MPa})^{4)}$ for the basal slip, indicating that the CRSS obtained in this study is reliable.

Here, no slip lines of b1 were observed on the surface since the slip direction of b1 is parallel to the surface of $\alpha$ specimen. Conversely, slip lines were clearly observed in Fig. 4(a). Thus, either b2 or b3 or both were activated. However, a shear force higher than that required for activation of $\mathrm{b} 1$ is required to activate either b2 or b3 due to differences in slip and shear direction. Therefore, further investigation is required to clarify why b2 and b3, either singularly or in tandem, were simultaneously activated with $\mathrm{b} 1$.

\section{$3.2 \beta$ specimen}

Figure 8 shows a typical shear stress - displacement curve of $\beta$ specimen. $\beta$ specimens yielded at shear yield stresses ranging from 5.5 $\mathrm{MPa}$ and $10 \mathrm{MPa}$, as shown in Table 2. The

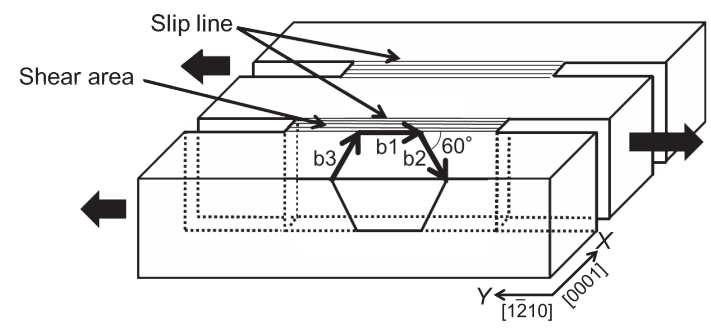

Fig. 7 Schematic illustration of basal slips with different slip directions in $\alpha$ specimen.

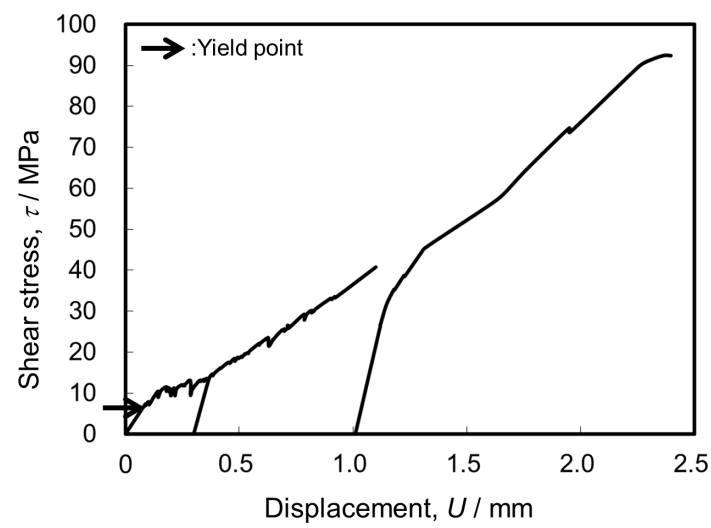

Fig. 8 Typical shear stress - displacement curve of $\beta 1$ specimen.
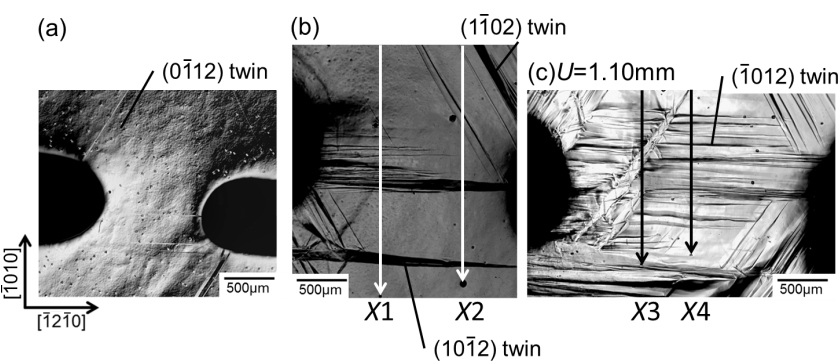

Fig. 9 Optical micrographs of shear areas at (a) $U=0.02 \mathrm{~mm}$ in $\beta 3$ specimen, (b) $U=0.37 \mathrm{~mm}$ and (c) $U=1.10 \mathrm{~mm}$ in $\beta 1$ specimen.

shear stress increased with small serration after yielding. Figure 9 shows optical micrographs of shear areas in $\beta$ specimen at (a) $U=0.02 \mathrm{~mm}$, (b) $U=0.37 \mathrm{~mm}$ and (c) $U=1.10 \mathrm{~mm}$. Here, $\{10 \overline{1} 2\}$ twins have six possible variants. Activated variants in this study were determined by observation of twin traces on (0001) and movement and directions of etch pits due to $\{10 \overline{1} 2\}$ twinning, described below. Also, two variants cannot be distinguished using twin traces on (0001) when the trace direction is the same. In this study, either of two indexes is used and shown in Fig. 9. Here, only three of six variants can be distinguished using twin traces observed on (0001). Either of two variants with the same trace is shown in this study. (0112) twins were observed outside the shear area immediately yielding at $U=0.02 \mathrm{~mm}$, as shown in Fig. 9 (a). (1012) twins parallel to the shear direction and (1102) twins were observed at $U=0.37 \mathrm{~mm}$ (Fig. 9 (b)). The shear area was filled with (1012) twins and (0112) twins at $U=1.10 \mathrm{~mm}$, as shown in Fig. 9 (c).

Prismatic slips activated in $\beta$ specimen made observation 
of slip lines impossible as slip direction is parallel to specimen surface. Therefore, in order to confirm activation of prismatic slip in $\beta$ specimen, displacement $d$ along the line $\mathrm{X} 1$, X2, X3 and X4 shown in Fig. 9(b) and (c) was measured as well in $\alpha$ specimen. Here, displacements vertical and parallel to the shear direction, $d x$ and $d y$, respectively occurred in $\mathrm{x}$ and y directions, due to $\{10 \overline{1} 2\}$ twinning in the shear area of $\beta$ specimen. The $d x$ and $d y$ were obtained by measuring movement of etch pits in $\mathrm{x}$ and $\mathrm{y}$ directions. Also, displacements vertical and parallel to the shear direction due to $\{10 \overline{1} 2\}$ twinning were defined as $d t x$ and $d t y$. The $d t x$ and $d t y$ were obtained using a shear strain per unit volume of a twin in each direction and widths of twins on $\mathrm{X} 1, \mathrm{X} 2, \mathrm{X} 3$ and $\mathrm{X} 4$ lines shown in Fig. 9 (b) and (c). Strains per unit volume of a twin

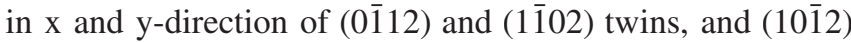
and $(\overline{1012})$ twins were theoritically calculated to be $4.3 \%$ and $7.4 \%$, and $8.6 \%$ and $0 \%$, respectively.

Figure 10(a) and (b) show changes in the $d y$ and dty, and $d x$ and $d t x$ on each line. The $d y$ and $d x$ were obtained using movement of etch pits at the shear displacement between $U=$ $1.10 \mathrm{~mm}$ and $0.37 \mathrm{~mm}$. Result revealed that $d x$ and $d y$ correspond to $d t x$ and $d t y$ in each, i.e., the shear area of $\beta$ specimen deformed solely by $\{10 \overline{1} 2\}$ twins. Therefore, prismatic slips were not activated in $\beta$ specimen at a shear stress of at least $40 \mathrm{MPa}$, at $U=1.10 \mathrm{~mm}$. Here, $d y$ and $U$ were much different, causing deformation outside the shear area of $\beta$ specimen. FEM were carried out to simulate shear stresses loading on $\{10 \overline{1} 2\}$ planes in order to investigate why $\{10 \overline{1} 2\}$ twins occurred in $\beta$ specimen.

Figure 11 (a) and (b) show illustrations of a pure shear test specimen and finite element model created on the basis of a half shape of the pure shear test specimen. In the present sim-
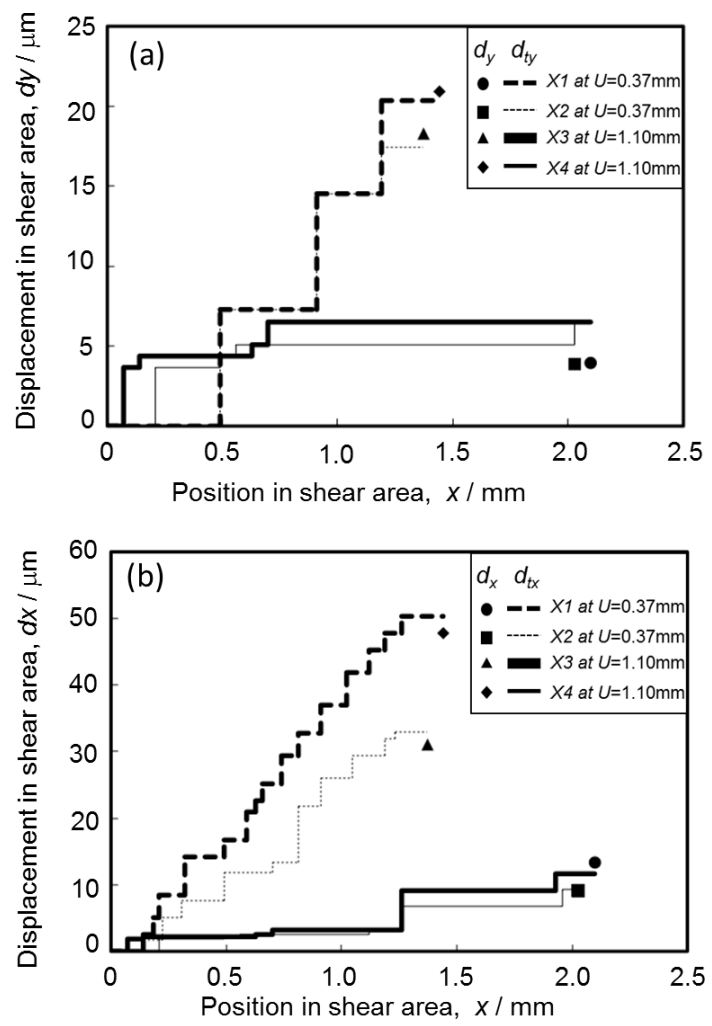

Fig. 10 Changes in (a) $d y$ and dty, and (b) $d x$ and $d t x$ on each line. ulation, load was applied to the lower half of the specimen indicated by (A) in the upper direction, as shown in Fig. 11 (b). Figure 12 shows the relationship between the shear stress on the prismatic plane $\tau$ and the shear stress in the twin direction on the $\{10 \overline{1} 2\}$ twin plane at each node; $\tau_{T}$ is shown in Fig. 11 (b). $\tau_{T}$ was calculated using the following equation.

$$
\tau_{T}=\left(\begin{array}{lll}
x_{1} & y_{1} & z_{1}
\end{array}\right)\left(\begin{array}{lll}
\sigma_{x} & \tau_{x y} & \tau_{x z} \\
\tau_{y x} & \sigma_{y} & \tau_{y z} \\
\tau_{z x} & \tau_{z y} & \sigma_{z}
\end{array}\right)\left(\begin{array}{l}
x_{2} \\
y_{2} \\
z_{2}
\end{array}\right)
$$

Where $\sigma_{x}, \sigma_{y}, \sigma_{z}$ are normal stresses in the $\mathrm{x}, \mathrm{y}, \mathrm{z}$ directions at each node, $\tau_{x y}, \tau_{y z}, \tau_{z x}$ are shear stresses in the $\mathrm{x}, \mathrm{y}, \mathrm{z}$ directions at each node. $x_{1}, y_{1}, z_{1}$ and $x_{2}, y_{2}, z_{2}$ were the slip direction and the vertical direction to the slip plane, respectively. Results of a slip plane with the highest $\tau_{T}$ are shown in Fig. 12, although the $\{10 \overline{1} 2\}$ twin has six equivalent twin planes. As a result, it was found that shear stress exceeding 2.5 MPa of CRSS for $\{10 \overline{1} 2\}$ twins were applied to twin planes at several nodes when a shear stress of $17 \mathrm{MPa}$ was applied to the prismatic plane. Also, directions of twin planes with the highest $\tau_{T}$ at each node are shown in Fig. 13. $\{10 \overline{1} 2\}$ twin variants can be distinguished due to their radically different directions (0001), as shown in Fig. 13 (a). Figure 13 (b) shows directions of twin planes with the highest $\tau_{T}$ at each node. Here, the magnitude of the $\tau_{T}$ is expressed by the length of the line. It was found that higher shear stresses operate on (1012) twin plane parallel to shear direction at the tip of notches; that is, the force in the normal direction of shear stress is loaded at the tip of the notches. Actual distribution of
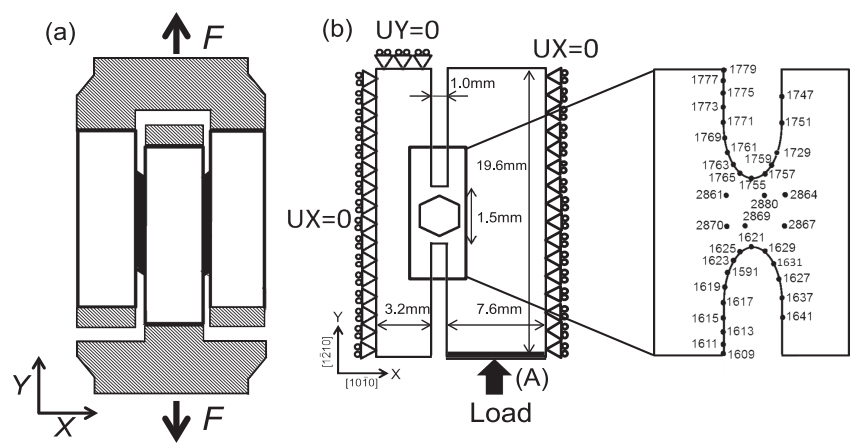

Fig. 11 Schematic illustrations of (a) pure shear test specimen and (b) finite element model created on the basis of a half shape of the pure shear test specimen.

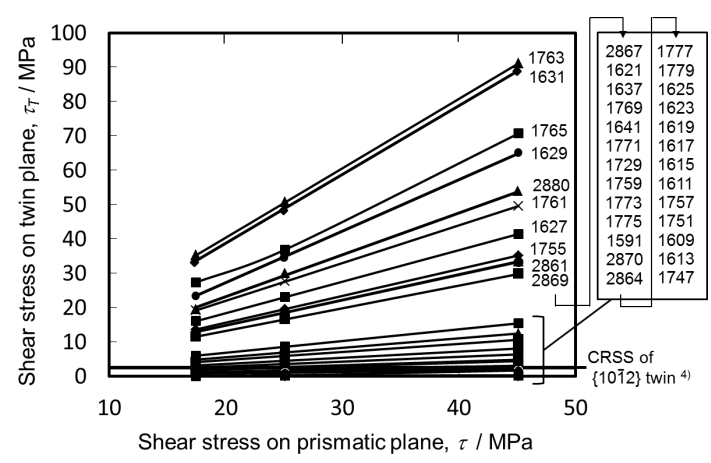

Fig. 12 Relation between shear stress on prismatic plane and shear stress on $\{10 \overline{1} 2\}$ twin plane at each node. 
(a)
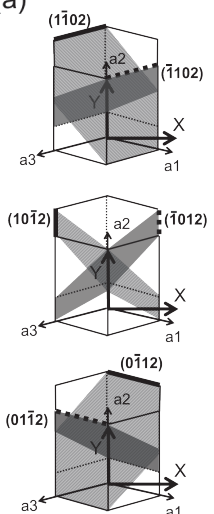

(b) $\tau=45 \mathrm{MPa}$

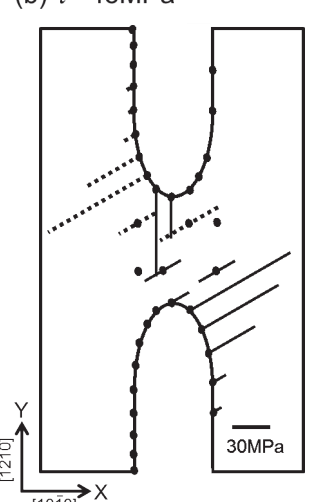

(c) $U=0.16 \mathrm{~mm}$

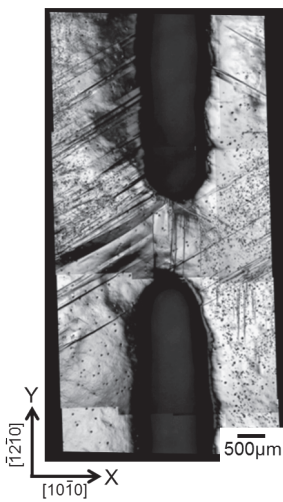

Fig. 13 Schematic of (a) $\{10 \overline{1} 2\}$ twin variants and (b) directions of twin planes with highest $\tau_{T}$ at each node, and (c) optical micrograph of shear area in $\beta 3$ specimen.

$\{10 \overline{1} 2\}$ twins on the surface of $\beta$ specimen shown in Fig. 13(c) was in a good agreement with the FEM result shown in Fig. 13(b). These results show that force was actually applied only to the lower half of the specimen indicated by (A) in Fig. 11 (b). Compressive stress was thus loaded to the specimen, and as a result, twinning occurred outside the shear area. Therefore, to investigate the activation of the prismatic slip, the present pure shear test requires improvement to measure application of shear force at only the shear area.

Consequently, it can be confirmed that no prismatic slips are activated at a shear stress of $40 \mathrm{MPa}$ in $\beta$ specimen from results shown in Fig. 10. Although measurement of displacement on the specimen surface is difficult with a shear stress of $90 \mathrm{MPa}$ applied to the prismatic plane, many twins can be observed. This indicates that the $\beta$ specimen deforms solely due to twins.

Twins occurring prior to slips $\beta$ specimen would also result in twin interfaces as obstacles for slips. As such, CRSS for slip systems is unable to be determined using the shear stress obtained in the present test. While prismatic slip can be confirmed not to activate at the shear stress of $40 \mathrm{MPa}$, this was unable to evaluate CRSS.

\section{Conclusion}

Pure shear tests of $\mathrm{Mg}$ single crystals were carried out to evaluate CRSSs for basal and prismatic slips. The main results are summarized as follows:

(1) Basal slips were activated in $\alpha$ specimen, and CRSS was estimated to be $0.7 \mathrm{MPa}$.

(2) Prismatic slips were not activated at a shear stress of $40 \mathrm{MPa}$ in $\beta$ specimen at least.

(3) $\{10 \overline{1} 2\}$ twins occurred in $\beta$ specimen and were also confirmed by the finite element method.

\section{Acknowledgement}

This study was supported by JSPS KAKENHI Grant Number 26420667 and "The Light Metal Educational Foundation Inc." in Japan.

\section{REFERENCES}

1) H. Yoshinaga and R. Horiuchi: Trans. JIM 5 (1964) 14-21.

2) H. Asada and H. Yoshinaga: J. Jpn. Inst. Metals 23 (1963) 67-71.

3) S. Ando, K. Nakamura, K. Takashima and H. Tonda: J. Japn Inst. Light Metals 42 (1992) 765-771.

4) R.L. Bell and R.W. Cahn: Proc. Roy. Soc. A 239 (1957) 494-521.

5) R. von. Mises and Z. Angew: Math. Mech. 8 (1928) 161-185.

6) K. Miyauchi : Sci. Papers RIKEN 78 (1984) 27-42. 